\title{
DDP4 inhibition reduces fibrosis
}

Tissue fibrosis and persistent activation of fibroblasts are hallmark features of systemic sclerosis (SSc). Findings from a new study highlight the serine protease dipeptidyl-peptidase-4 (DPP4, also known as CD26) as not only a marker of activated fibroblasts in SSc, but also as a potential therapeutic target for treating fibrosis in SSc.

Inhibitors of DPP4 (such as sitagliptin) are already approved and widely used for the treatment of diabetes mellitus owing to the ability of DPP4 to target and inhibit incretin hormones. However, DPP4 also has a broad range of other substrates, including chemokines, and can also function independently of its enzymatic activity, bestowing DPP4 with a broad range of functions.

In these latest findings, the investigators found that the expression of DPP4 and the number of DPP4expressing fibroblasts is upregulated in the skin of patients with SSc compared with the skin of healthy individuals.
Incubation of human dermal fibroblasts with recombinant transforming growth factor- $\beta$ (TGF $\beta$ ), a key profibrotic cytokine in SSc, induced the expression and enzymatic activity of DDP4 in vitro. This upregulation was dependent on non-canonical TGF $\beta$ signalling via the kinase ERK.

In fibroblasts from either mice or humans, the expression of DDP4 was associated with increased expression of myofibroblast markers and type I collagens as well as with increased responsiveness of the fibroblasts to stimulation with TGF $\beta$. Notably, treatment of fibroblasts from patients with SSc with sitagliptin inhibited TGF $\beta$-induced fibroblast-tomyofibroblast transition and release of type I collagens.

Loss of DPP4 activity through gene knockout or treatment with sitagliptin ameliorated disease in mice with bleomycin-induced dermal or pulmonary fibrosis. Importantly, treatment could also induce regression of pre-established fibrosis

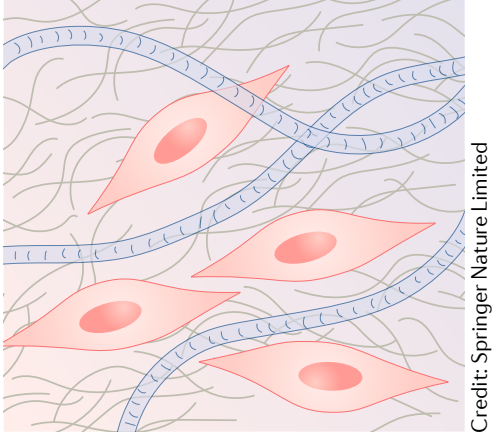

in mice with bleomycin-induced fibrosis as well as in mice with graft-versus-host disease.

The authors speculate that a subpopulation of DPP4-positive fibroblasts promote tissue fibrosis in SSc, and they plan to use lineage tracing experiments to confirm this theory. They also plan to use additional experimental models to further confirm the antifibrotic effects of this enzyme, with the long-term aim of a clinical trial in SSc.

Jessica McHugh with sitagliptin ameliorated disease

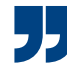

\section{Placental growth factor links angiogenesis and autoimmunity}

Increased angiogenesis and immune cell infiltration go hand-in-hand at sites of inflammation in autoimmune diseases, such as rheumatoid arthritis (RA). The results of a new study suggest that placental growth factor (PIGF), a vascular endothelial growth factor homologue, could help to mediate both processes by stimulating angiogenesis and Thelper $17\left(\mathrm{~T}_{\mathrm{H}} 17\right)$ cell differentiation.

"Although angiogenesis and T cell infiltration are tightly interwoven processes in both health and disease, it has not been clear whether angiogenic factors affect $T$ helper cell

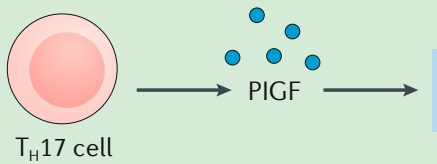

- Angiogenesis

- $T_{H} 17$ cell differentiation

Credit: Springer Nature Limited differentiation or whether a specific Thelper cell subset directly contributes to pathologic angiogenesis in autoimmune diseases," explains corresponding author Wan-Uk Kim.

To address this uncertainty, Kim and colleagues investigated the potential role of PIGF in crosstalk between endothelial cells and T cells. In vitro, PIGF was secreted specifically by activated $\mathrm{T}_{\mathrm{H}} 17$ cells, and $T$ cell-secreted PIGF could stimulate neovascularization both in vitro and in vivo.

The addition of PIGF-conditioned media or recombinant PIGF to $\mathrm{CD}^{+}$ T cells caused upregulation of the $T_{H} 17$ cell-specific transcription factor ROR $\gamma$. PIGF-mediated ROR $\gamma$ upregulation required the phosphorylation of signal transducer and activator 3 , similar to IL-6-mediated signalling during classical $T_{H} 17$ cell differentiation. Interestingly, PIGF could stimulate $\mathrm{T}_{H} 17$ cell differentiation in the absence of IL-6.

Placing these findings in the context of autoimmune disease, overexpression of PIGF in T cells exacerbated disease in mice with collagen-induced arthritis. PIGF concentrations also correlated with IL-17 concentrations in synovial fluid from patients with RA.

"Our findings provide novel insights into PIGF-mediated links between angiogenesis, $T_{H} 17$ cell development and autoimmunity, indicating that PIGF inhibitors might be able to control autoimmune and inflammatory diseases via the dual inhibition of angiogenesis and $\mathrm{T}_{\mathrm{H}} 17$ cell generation," says Kim.

The researchers are currently looking to develop such PIGF inhibitors for use in diseases with $T_{H} 17$ cell involvement, such as RA.

Joanna Collison

ORIGINAL ARTICLE Yoo, S.-A. et al. Placental growth factor regulates the generation of $\mathrm{T}_{\mathrm{H}} 17$ cells to link angiogenesis with autoimmunity. Nat. Immunol. https://doi.org/10.1038/s41590019-0456-4 (2019) 